\title{
MicroRNA-21-5p induces the metastatic phenotype of human cervical carcinoma cells in vitro by targeting the von Hippel-Lindau tumor suppressor
}

\author{
LINA CAI $^{1}$, WULIANG WANG ${ }^{1}$, XIAOMEI LI ${ }^{1}$, TIELI DONG ${ }^{2}$, QING ZHANG $^{1}$, \\ BAOJV ZHU ${ }^{1}, \mathrm{HU}^{2} \mathrm{ZHAO}^{1}$ and SHUBIAO WU ${ }^{2}$ \\ Departments of ${ }^{1}$ Obstetrics and Gynecology, and ${ }^{2}$ Anesthesiology, The Second Affiliated \\ Hospital of Zhengzhou University, Zhengzhou, Henan 450014, P.R. China
}

Received November 29, 2016; Accepted September 15, 2017

DOI: $10.3892 / 01.2018 .7937$

\begin{abstract}
Numerous studies have indicated that microRNAs (miRs), a group of small non-coding RNAs, are determining regulatory elements involved in the pathogenesis of various types of cancer, including cervical cancer (CC). Although miR-21-5p upregulation has been demonstrated to associate with tumorigenesis by controlling the expression of oncogenic and tumor suppressor genes, only a small number of studies have investigated the expression of miR-21-5p and its functional role in CC. The objective of the present study was to investigate the effect of miR-21-5p on the proliferation, apoptosis, migration and invasion of $\mathrm{CC}$ cells, and the potential underlying molecular mechanism of these effects. The measurement of miR-21-5p levels using quantitative polymerase chain reaction revealed that miR-21-5p was markedly increased in CC cell lines compared with normal cells. Upon silencing of miR-21-5p, a marked suppression of the proliferation, migration and invasion of CaSki cells was observed, with induction of cell apoptosis. These effects were reversed with miR-21-5p overexpression. A database search followed by a luciferase reporter assay ascertained that the 3'-untranslated region of the von Hippel-Lindau tumor suppressor (VHL) mRNA sequence was a direct target of miR-21-5p. Furthermore, silencing of VHL neutralized the effects of miR-21-5p inhibition. These observations suggested that miR-21-5p is an oncogene that is able to promote the metastatic phenotype of $\mathrm{CC}$ cells through downregulation of VHL expression, which may present a path to novel therapeutic stratagems for the CC therapy.
\end{abstract}

Correspondence to: Dr Shubiao Wu, Department of Anesthesiology, The Second Affiliated Hospital of Zhengzhou University, 2 Jingba Road, Jinshui, Zhengzhou, Henan 450014, P.R. China

E-mail: wushubiao160379@163.com

Key words: microRNA-21, cervical carcinoma, proliferation, invasion, apoptosis, von Hippel-Lindau

\section{Introduction}

As the third most commonly occurring cancer type in females, cervical cancer (CC) is the fourth leading cause of cancer-associated mortality globally, resulting in $\sim 300,000$ mortalities each year (1-3). In previous years, diagnosis and treatment options for CC have been markedly improved; however, CC remains a public health concern worldwide due to the lack of detailed data on the molecular mechanisms underlying its pathogenesis. There is therefore an urgent requirement to uncover novel molecular diagnostic markers and therapeutic targets for the treatment of CC.

MicroRNAs (miRNAs/miRs) are a family of small non-coding RNA molecules that are able to bind to the 3'-untranslated region (3'-UTR) of specific mRNA targets to inhibit their translation. Several previous studies have demonstrated that miRNAs are typically deregulated or induce the abnormal expression of key genes and pathways governing cancer-associated processes $(4,5)$.

Previous studies have demonstrated that miR-21-5p is upregulated in numerous types of cancer and has been associated with cellular proliferation, migration, invasion and apoptosis, which are the main processes underlying cancer pathogenesis $(6,7)$. It has been demonstrated that the downregulation of special AT-rich sequence-binding protein 1 and miR-21-5p overexpression in clear cell renal cell carcinoma are associated with each other, and with poor prognosis (8). A prior study demonstrated the association of miR-21-5p overexpression with tongue cancer cell chemo-resistance via cell adhesion molecule 1 targeting (9). miR-21-5p overexpression has also been identified as a possible predictor of tumor regression following chemoradiotherapy in patients with rectal cancer (10), and as a biomarker of recurrence in young patients with gastric cancer (11) and thymic epithelial tumors (12). It has been reported that the competing endogenous regulation of miR-21-5p by the long non-coding RNA maternally expressed 3 inhibits CC cell proliferation and apoptosis (13). miR-21-5p dysregulation has shown promise as a biomarker for the diagnosis of CC (7). At the molecular level, it has been demonstrated that miR-21-5p promotes $\mathrm{CC}$ cell proliferation and migration by inhibiting phosphatase and tensin homolog 
(PTEN) (3). However, the underlying molecular mechanisms of miR-21-5p in these processes in CC remain unresolved.

Typically, miRNAs execute their function by targeting tumor suppressor genes or oncogenes. To fully appreciate the implication of miR-21-5p in CC pathogenesis, it is important to study its association with these molecules. Among the tumor suppressor genes, von Hippel-Lindau tumor suppressor (VHL), which is located on chromosome $3 \mathrm{p} 25$, is downregulated due to mutations or silencing in the majority of carcinomas (14-16). This downregulation of VHL induces hypoxia via hypoxia-inducible factors (HIF), and subsequent angiogenesis and cell growth (14-16). The VHL-dependent regulation of miRNAs in renal cancer has been previously studied and results indicated that miR-21-5p may be regulated by VHL. Thus far, the association between miR-21-5p and VHL remains unresolved.

The objective of the present study was therefore to verify whether miR-21-5p and VHL interact with each other and serve a function in $\mathrm{CC}$, and to uncover the molecular mechanisms underlying these processes in $\mathrm{CC}$.

\section{Materials and methods}

Cell line and cell culture. Human cervical carcinoma SiHa, HeLa, CaSki, c4-1 and c-33a cell lines and normal cervical Ect1/E6E7 cells were provided by the American Type Culture Collection (Manassas, VA, USA). Dulbecco's modified Eagle's medium (DMEM; Thermo Fisher Scientific, Inc., Waltham, MA, USA) supplemented with $10 \%$ bovine calf serum (Gibco; Thermo Fisher Scientific, Inc.) was used for cell culture. All cells were cultured at $37^{\circ} \mathrm{C}$ in a humidified incubator with $5 \%$ $\mathrm{CO}_{2}$.

Transfection. The miR-21-5p mimic (catalog no. HMI0371; Mission ${ }^{\circledR}$ ) (sense, 5'-UAGCUUAUCAGACUGAUGUUG AA-3', and antisense, 5'-UCAACAUCAGUCUGAUAAGCU AUU-3'), negative control (NC) (sense, 5'-UUCUCCGAACGU GUCACGUTT-3', and antisense, 5'-ACGUGACACGUUCGG AGAATT-3'), miR-21-5p inhibitor (catalog no. HLTUD0371; Mission; 5'-CUUCAACAUCAGUCUGAUAAGCUA-3') were purchased from Sigma-Aldrich (Merck KGaA, Darmstadt, Germany). The human VHL small interfering (si)RNAs (cat no. CRH5082-30) (A, sense, 5'-CCAGGUCAUCUUCUG CAAUTT-3', and antisense, 5'-AUUGCAGAAGAUGACCUG GTT-3'; B, sense, 5'-GGGCUUCUGGUUAACCAAATT-3', and antisense, 5'-UUUGGUUAACCAGAAGCCCTT-3'; C, sense, 5'-GGAGCGCAUUGCACAUCAATT-3', and antisense, 5'-UUGAUGUGCAAUGCGCUCCTT-3') were purchased from Cohesion Biosciences (London, UK). The siRNAs A, $\mathrm{B}$ and $\mathrm{C}$ were transfected at an equimolar ratio. A total of 5,000 CaSki cells were seeded in 96-well plates and once cells had grown to $50-80 \%$ confluence, they were transfected with miR-21-5p mimic (10 nM) or transduced with the miR-21-5p inhibitor $(10 \mathrm{nM})$ following the procedures described in the manufacturer's protocol, while VHL siRNA (100 nM) was transfected with Lipofectamine ${ }^{\circledR} 2000$ (Invitrogen; Thermo Fisher Scientific, Inc.) according to the manufacturer's protocol. Cells were harvested after $72 \mathrm{~h}$ for subsequent analysis.

Reverse transcription-quantitative polymerase chain reaction $(R T-q P C R)$. For the analysis of VHL mRNA expression, total
RNA was extracted from CC cell lines and normal cervical cells using TRIzol reagent (Invitrogen; Thermo Fisher Scientific, Inc.) and reverse transcribed using the Invitrogen SuperScript ${ }^{\mathrm{TM}}$ III Reverse Transcriptase kit (Thermo Fisher Scientific, Inc.) according to the manufacturer's protocol. For miR-21-5p expression analysis, the mirVana miRNA Isolation kit (Thermo Fisher Scientific, Inc.) was used according to the manufacturer's protocol and cDNA was generated with miRNA Reverse Transcription kit (Qiagen China Co., Ltd., Shanghai, China). qPCR was performed using StepOnePlus Real-Time PCR system (Thermo Fisher Scientific, Inc.) with The PrimePCR ${ }^{\mathrm{TM}}$ Template for $\mathrm{SYBR}^{\circledR}$ Green assay (VHL, Human) primers (sense, 5'-GAGATGCAGGGACACACGAT-3', and antisense, 5'-ATCCGTTGATGTGCAATGCG-3'; internal reference GAPDH, sense, 5'-GCCAGTAGAGGCAGGGATGATGTT C-3', and antisense, 5'-CCATGTTCGTCATGGGTGTGAACC A-3') (Bio-Rad Laboratories, Inc., Hercules, CA, USA) for VHL PCR reaction and MystiCq ${ }^{\circledR}$ microRNA qPCR Assay Primer hsa-miR-21-5p (Sigma Aldrich; Thermo Fisher Scientific, Inc.) for the miR-21-5p PCR reaction. GAPDH and U6 small nuclear RNA were used for the normalization of VHL and miR-21-5p levels, respectively. Relative expression was estimated based on the $2^{-\Delta \Delta \mathrm{Cq}}$ method (17).

Cell viability assay. Proliferation of CaSki cells was measured using MTT assay. Cells were transfected for $24 \mathrm{~h}$ then seeded in 96-well microtiter plates for $72 \mathrm{~h}\left(1 \times 10^{4}\right.$ cells/well). MTT was added for another $4-\mathrm{h}$ incubation at $37^{\circ} \mathrm{C}$. The medium was aspirated and $150 \mu \mathrm{l}$ DMSO was added for $15 \mathrm{~min}$ until the crystals were dissolved. The absorbance was determined using spectrophotometry at $490 \mathrm{~nm}$ using a microplate reader. The experiments were repeated in triplicate.

Cell migration and invasion assays. To determine their invasion and migration abilities CaSki cells, which expressed a higher level of miR-21-5p than the other examined cell lines, were cultured in Matrigel-coated (invasion) or uncoated (migration) Transwell inserts. Cells transfected with miR-21-5p mimic/inhibitor or VHL inhibitor or controls $\left(\sim 2 \times 10^{4}\right.$ cells) were suspended in $200 \mathrm{ml}$ serum-free DMEM and seeded in the upper chamber. In the lower chamber, the DMEM was supplemented with bovine calf serum (Gibco; Thermo Fisher Scientific, Inc.) and used as a chemo-attractant. After $48 \mathrm{~h}$ of incubation, the cells that had migrated through the Matrigel-coated or uncoated membrane were fixed in $100 \%$ methanol for $10 \mathrm{~min}$, stained with $0.1 \%$ crystal violet for $10 \mathrm{~min}$ at room temperature, and counted under a white light microscope (Olympus, Tokyo, Japan).

Western blot analysis. CaSki cells were lysed with radioimmunoprecipitation assay lysis buffer (Beyotime Institute of Biotechnology, Haimen, China). Protein concentrations were determined by a BCA assay. Protein samples (20 $\mu \mathrm{g}$ per lane) were separated using $12 \%$ SDS-PAGE and transferred to a PVDF membrane (Bio-Rad Laboratories, Inc.). The membrane was blocked with skimmed milk solution (10\%) in TBST at room temperature and incubated overnight at $4{ }^{\circ} \mathrm{C}$ with VHL antibody at dilution of 1:3,000 (catalog no. FL-181; Santa Cruz Biotechnology, Inc., Dallas, TX, USA), caspase-3 at dilution of 1:500 and cleaved caspase-3 antibodies at dilution of 1:2,000 
(catalog no. Asp175; Cell Signaling Technology, Inc., Danvers, MA, USA). Following washing with TBST buffer (three x15 min), the appropriate horseradish peroxidase-conjugated goat anti-rabbit secondary antibody at a dilution of 1:4,000 (Invitrogen; Thermo Fisher Scientific, Inc.; catalog no. 65-6120), was added for $1 \mathrm{~h}$ at room temperature. $\beta$-actin antibody at a dilution of 1:2,000 was used as an internal reference (Cell Signaling Technology, Inc.; catalog no. 4970). The immunoreactive proteins were visualized using an enhanced chemiluminescence system (GE Healthcare Life Sciences, Little Chalfont, UK) and grayscale value analysis by ImageJ software was used for quantification.

Caspase-Glo 3/7 assay. CaSki cells were transfected for $24 \mathrm{~h}$ then seeded in 96-well microliter plates for $48 \mathrm{~h}$ (1x10 4 cells/well). The 96-well plates containing transfected cells were removed from the incubator and were allowed to equilibrate to room temperature. A total of $100 \mu \mathrm{l}$ Caspase-Glo ${ }^{\circledR}$ 3/7 reagent (Promega Corporation, Madison, WI, USA) was then added to each well of a white-walled 96-well plate containing $100 \mu \mathrm{l}$ of culture medium containing untransfected cells, transfection control cells or transfected cells in culture medium. The contents of the wells were mixed using a plate shaker at $300 \mathrm{rpm}$ for $30 \mathrm{sec}$, and incubated at room temperature for $1 \mathrm{~h}$. The luminescence of each sample was measured using a plate-reading luminometer.

Luciferase reporter assay. The miR-21-5p binding sites from the 3'-UTR of VHL or mutant 3'-UTR were cloned into the pGL3 reporter luciferase vector (Shanghai GeneChem Co., Ltd., Shanghai, China). CaSki cells were cultured in 96-well plates. Aliquots of $10 \mathrm{nM}$ miR-21-5p mimic or control miRNA were cotransfected with $0.1 \mathrm{mg}$ of the pGL3-3' UTR wild-type or mutant plasmid DNA into CaSki cells using Lipofectamine 2000 (Invitrogen; Thermo Fisher Scientific, Inc.). Following transfection for $48 \mathrm{~h}$, luciferase activity was measured using the Dual-Luciferase Reporter Assay system according to the manufacturer's protocol (Promega Corporation). The relative luciferase activity was normalized against Renilla luciferase activity. The experiments were conducted in triplicate.

Bioinformatics prediction. TargetScan Human 7.0 (http://www .targetscan.org/) was used to identify potential targets of miR-21-5p.

Statistical analysis. All statistical analyses were conducted using GraphPad Prism software (version 6; GraphPad Software, Inc., La Jolla, CA, USA). The data are expressed as the mean \pm standard deviation. Differences between groups were assessed using a one-way analysis of variance (ANOVA) followed by the Bonferroni multiple comparison post-hoc test or using a two-way ANOVA with the Bonferroni multiple comparison post-hoc test. $\mathrm{P}<0.05$ was considered to indicate a statistically significant difference.

\section{Results}

miR-21-5p is upregulated in CC cell lines. In order to analyze the effect of miR-21-5p on the progression of $\mathrm{CC}$, the expression level of miR-21-5p was measured in CC cell lines. As

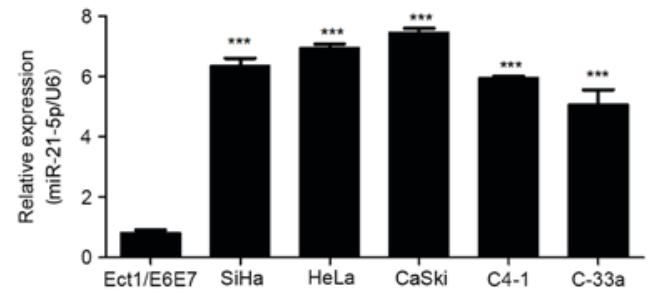

Figure 1. Expression of miR-21-5p is upregulated in CC cell lines. The expression of miR-21-5p in CC cells, as determined using reverse transcription-quantitative polymerase chain reaction. Statistical analysis was performed using one-way analysis of variance. U6 was used as an endogenous control. Error bars represent the standard deviation. ${ }^{* * *} \mathrm{P}<0.001$ vs. Ect1/E6E7. miR, microRNA; CC, cervical cancer.

presented in Fig. 1, miR-21-5p expression was significantly upregulated in CC SiHa, HeLa, CaSki, C4-1 and C-33a cell lines compared with that in the normal cervical cells. This suggests that miR-21-5p may be involved in the development of $\mathrm{CC}$.

Overexpression of miR-21-5p suppresses cell proliferation, migration and invasion, and induces apoptosis in vitro. To further analyze the effect of miR-21-5p on the proliferation, migration and invasion of CC cells, miR-21-5p mimic or inhibitor was transfected into CaSki cells. Compared with untransfected cells, the transfection of miR-21-5p mimic led to significant upregulation of miR-21-5p in CaSki cells, while miR-21-5p inhibitor led to significant repression of miR-21-5p (Fig. 2A).

The result of the MTT assay revealed that inhibition of miR-21-5p significantly suppressed the proliferation of the CaSki cells compared with untransfected cells, while the overexpression of miR-21-5p increased the proliferation of the CaSki cells (Fig. 2B). To evaluate the effect of miR-21-5p on the apoptosis of the CaSki cells, a caspase $3 / 7$ activity assay was performed and the results of the assay revealed that when cells were transfected with miR-21-5p inhibitor, the combined activity of Caspase 3 and Caspase 7 was significantly increased compared with that of untransfected cells, while the overexpression of miR-21-5p significantly decreased this activity (Fig. 2C). These results suggest that the inhibition of miR-21-5p was followed by induction of CaSki cell apoptosis. Furthermore, Transwell assays were used to measure the effect of miR-21-5p on cell migration and invasion. Compared with the untransfected control, the results revealed that the migration and invasion of CaSki cells were significantly suppressed when cells were transfected with miR-21-5p inhibitor, but increased when cells were transfected with miR-21-5p mimic, compared with the untransfected control (Fig. 2D and E). These results demonstrated that the inhibition of miR-21-5p expression suppressed CC cell proliferation, migration and invasion, and induced apoptosis in vitro.

miR-21-5p directly targets the VHL gene. VHL was predicted as the target gene of miR-21-5p using TargetScan Human 7.0 software (Fig. 3A). The expression of VHL in CC cells and normal cervical cells was measured. The results revealed that VHL expression was significantly decreased in all CC 


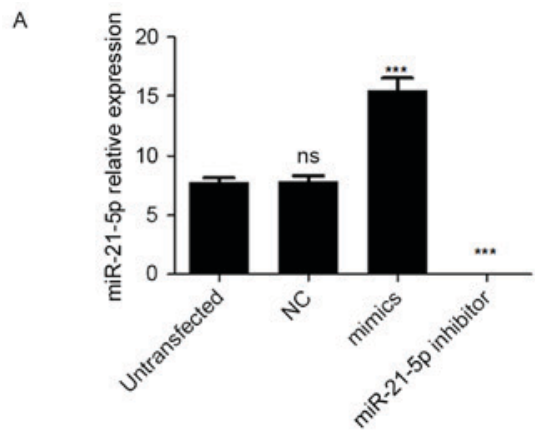

D

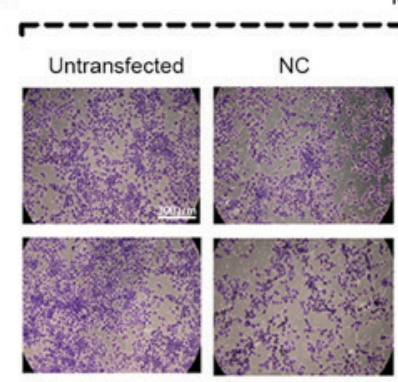

miR-21-5p mimic miR-21-5p inhibitor

Migration
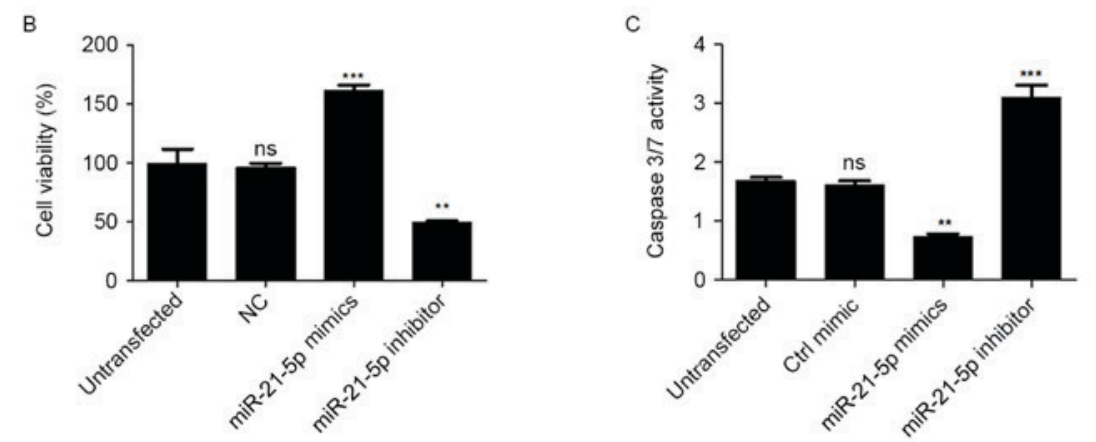

E

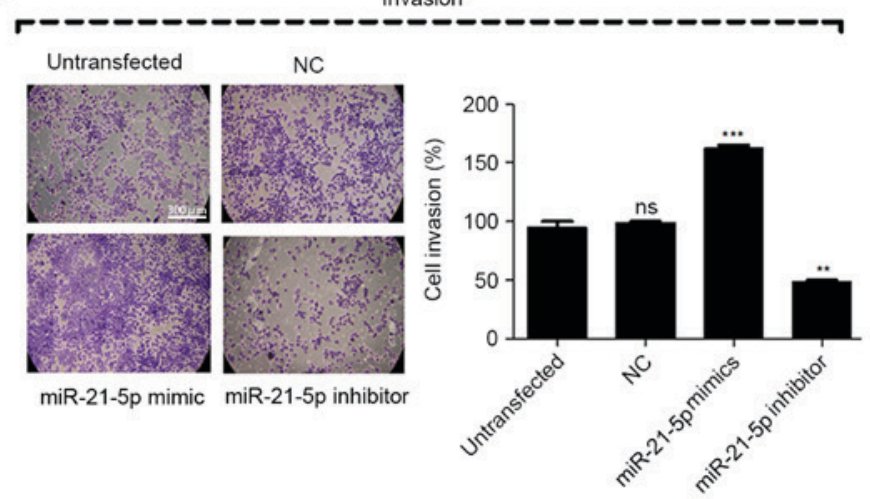

Figure 2. miR-21-5p induces the metastatic phenotype of the CC CaSki cell line. (A) miR-21-5p expression in CaSki cells transfected with miR-21-5p mimic/inhibitor. (B) miR-21-5p induces the proliferation of CaSki cells. (C) miR-21-5p inhibits CaSki cell apoptosis. (D) miR-21-5p induces the migration of CaSki cells. (E) miR-21-5p induces the invasion of CaSki cells. The expression of miR-21-5p in CC cells was determined using quantitative polymerase chain reaction, while Transwell chambers were used for determining the migration and invasion abilities of CaSki cells. Statistical analysis was performed using one-way analysis of variance. Error bars represent the standard deviation. ${ }^{* *} \mathrm{P}<0.01$ and ${ }^{* * *} \mathrm{P}<0.001$ vs. untransfected. ns, non-significant; miR, microRNA; $C C$, cervical cancer; NC, negative control; ctrl, control. Figures were obtained at x100 magnification of under a white light microscope following crystal violet staining.

A
Position 1689-1695 of VHL 3' UTR
$5^{\prime}$
... UUgCuCUAUGUUAGUAUAAGCUU. . .
hsa-miR-21-5p
3'
AGUUGUAGUCAGACUAUUCGAU

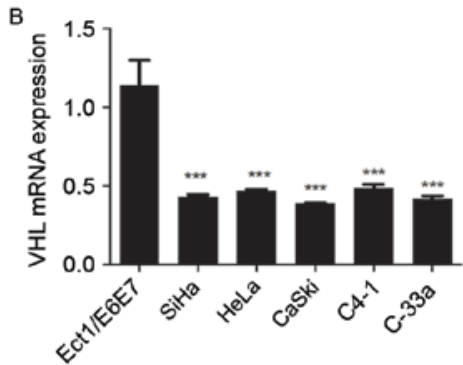

C
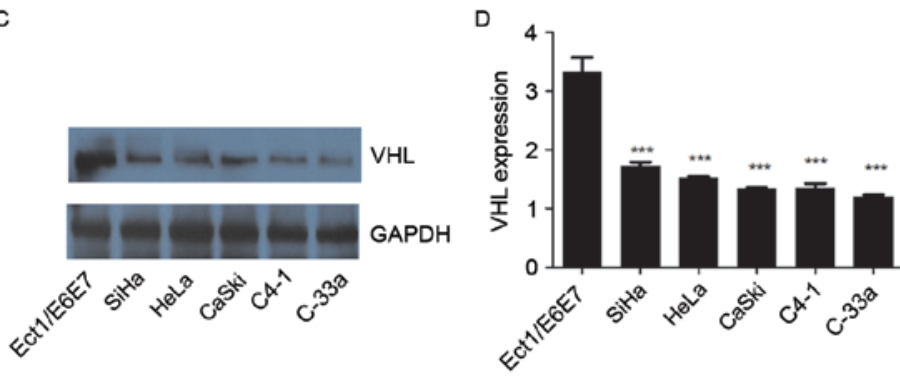

E
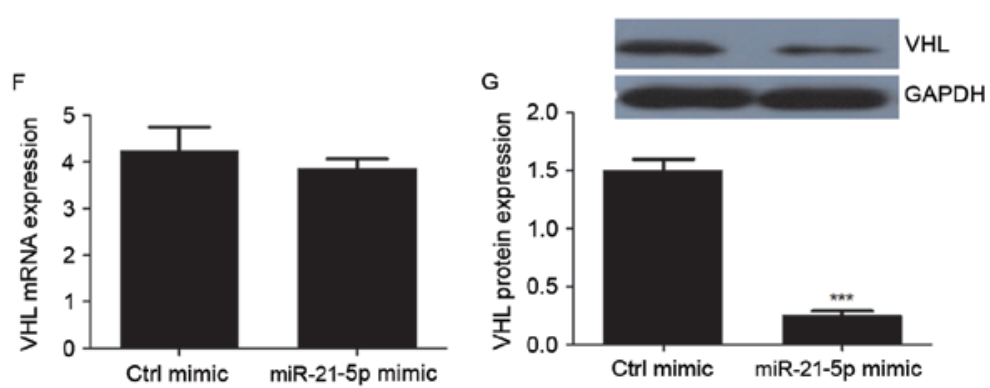

Figure 3. VHL, downregulated in CC, is a direct target of miR-21-5p. (A) Targetscan prediction of miR-21-5p binding site on the 3'-UTR region of VHL. (B) mRNA expression of VHL in CC cells. (C) Western blot analysis of VHL in different CC cell lines. (D) Quantitative representation of VHL in different CC cell lines. GAPDH was used as endogenous control. (E) Luciferase reporter assay for VHL. (F) Effect of miR-21-5p expression on the mRNA expression of VHL in CaSki cells. (G) Effect of miR-21-5p expression on the protein expression of VHL in CaSki cells. Statistical analysis was performed using one-way ANOVA or two-way ANOVA (for luciferase activity assay). Error bars represent the standard deviation. ${ }^{* * *}$ P $<0.001$ compared with Ect1/E6E7 or MUT groups. ns, non-significant; CC, cervical cancer; miR, microRNA; VHL, von Hippel-Lindau suppressor; ANOVA, analysis of variance; WT, wild-type; MUT, mutant; ctrl, control; UTR, untranslated region. 
A

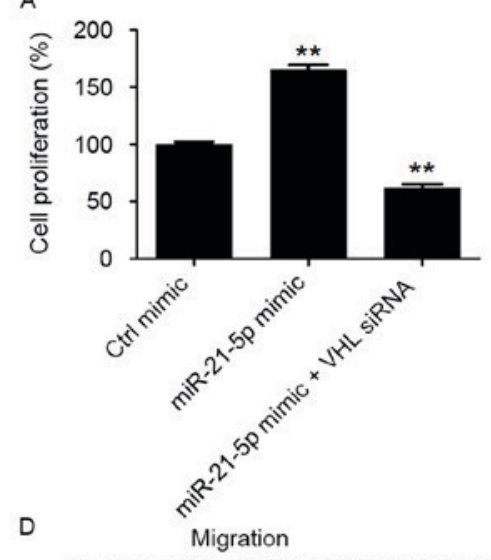

B

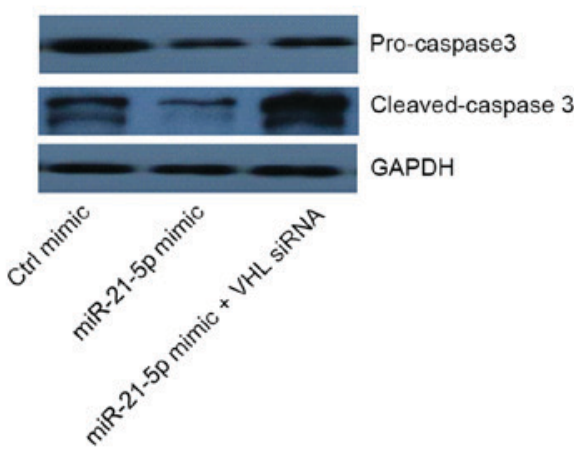

Invasion
C
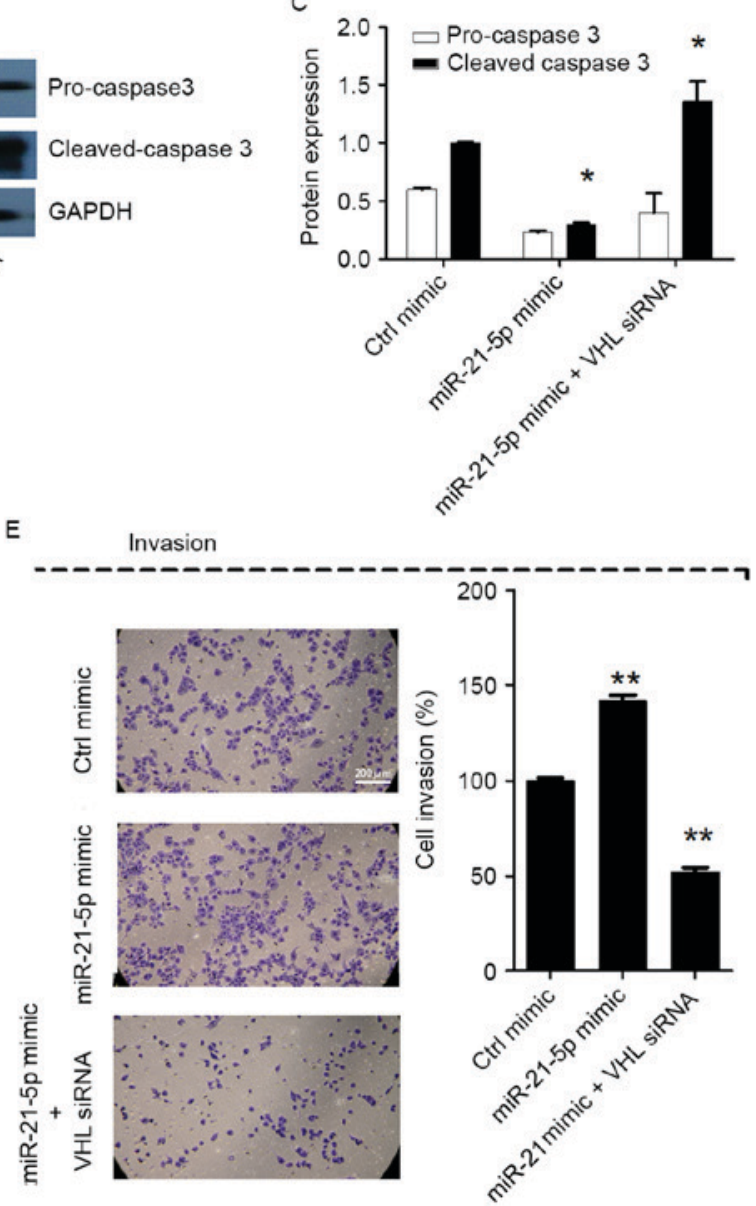

Figure 4. miR-21-5p induces the metastatic phenotype of the CC CaSki cell line via targeting VHL. (A) Silencing of VHL counteracted the effect of miR-21-5p on the proliferation of CaSki cells. (B) Western blot analysis of caspase 3 and cleaved-caspase 3 in different CaSki cells with GAPDH used as an endogenous control. (C) Silencing of VHL counteracted the effect of miR-21-5p on the pro-caspase 3 and cleaved caspase 3 activity in CaSki cells. (D) Silencing of VHL counteracted the effect of miR-21-5p on the migration of CaSki cells. (E) Silencing of VHL abrogated the effect of miR-21-5p on the invasion of CaSki cells. Cell images were obtained at x100 magnification under a white light microscope following crystal violet staining. The cell proliferation was measured using an MTT assay, while Transwell chambers were used for determining the migration and invasion abilities of CaSki cells. Statistical analysis that ctrl mimics were used for control was performed using one-way ANOVA or two-way ANOVA. Error bars represent the standard deviation. " $\mathrm{P}<0.05$ and ${ }^{* * *} \mathrm{P}<0.01$ vs. Ctrl mimic group. ns, non-significant; ANOVA, analysis of variance; miR, microRNA; CC, cervical cancer; VHL, von Hippel-Lindau suppressor; miR-21, miR-21-5p; siRNA, small interfering RNA; Ctrl, control.

cell lines compared with the controls (Fig. 3B-D). To further confirm whether VHL is a target of miR-21-5p, a dual luciferase assay was performed. The results revealed that the miR-21-5p mimic significantly increased luciferase activity in the mutant cells compared with that in the wild-type cells. Treatment with an miR-21-5p inhibitor produced no significant difference in luciferase activity between wild-type and mutant cells (Fig. 3E). The results of qPCR identified that the expression of VHL mRNA was not significantly altered following overexpression of miR-21-5p in the CaSki cells (Fig. 3F). Furthermore, overexpression of miR-21-5p resulted in downregulation of VHL protein expression $(\mathrm{P}<0.05$; Fig. 3G). These results suggest that VHL is a direct target gene of miR-21-5p.

Silencing of miR-21-5p suppresses cell proliferation, migration and invasion, and induces apoptosis by targeting VHL in $C C$. In order to analyze whether miR-21-5p promotes cell proliferation, migration and invasion, and inhibits apoptosis by targeting VHL, miR-21-5p mimic and VHL siRNA were cotransfected into CaSki cells. The results revealed that when cells were co-transfected with miR-21-5p mimic and VHL siRNA, the cell proliferation, migration and invasion were markedly decreased, and cell apoptosis was increased, compared with the group transfected with miR-21-5p mimic only $(\mathrm{P}<0.05$; Fig. 4A-E). The results indicated that VHL siRNA counteracted the effect of miR-21-5p on CaSki cells. Taken together, these results suggest that silencing of miR-21-5p suppresses cell proliferation, migration and invasion, and induces apoptosis by upregulating VHL in CC cells.

\section{Discussion}

Current therapies for managing CC, one of the major mortality factors in the female population, are typically unsuccessful due to the invasive and metastatic phenotype of CC cells. Previous studies have demonstrated that miR-21-5p is upregulated in the pathogenesis of CC and other tumors $(3,7,10,11,13)$. Parallel studies have equally demonstrated loss or downregulation of 
VHL in several types of cancer, particularly during oxidative stress, one of the factors initiating and controlling cancer progression and metastasis (14-16,18-22).

In the present study, the expression patterns of miR-21-5p and VHL were assessed in CC cell lines. The results demonstrated that miR-21-5p was upregulated in CC cell lines compared with normal control cells. Additionally, as cell viability and the occurrence of invasion and distant metastasis are important factors that affect the prognosis and treatment of CC, it was hypothesized that the upregulation of miR-21-5p may be involved in the proliferation and metastasis of CC cells. Therefore, the effect of miR-21-5p on the cell proliferation, migration, invasion and apoptosis of CaSki cells was investigated. The results revealed that inhibition of miR-21-5p expression significantly suppressed CaSki cell proliferation, migration and invasion, and induced cell apoptosis. This suggests that miR-21-5p may be associated with the progression of CC malignancy. The results were corroborated by previous studies, which produced similar results (3,9-12). Notably, it was identified that miR-21-5p stimulates the proliferation and migration of $\mathrm{CC}$ cells through inhibition of the PTEN pathway (3). However, the underlying molecular mechanism of miR-21-5p in cancer, particularly CC, remains unresolved.

Therefore, the objective of the present study was to explore the association between miR-21-5p and VHL. The expression level of VHL in CC cells was measured and it was revealed that unlike miR-21-5p, the expression of VHL was downregulated in the CC cell lines relative to the normal cells. Using bioinformatics a putative binding site for miR-21-5p was identified in the 3'-UTR region of VHL, which was validated as a direct target for miR-21-5p by luciferase assay. Overexpression of miR-21-5p significantly decreased the expression of VHL and the activity of the VHL 3'-UTR. Furthermore, miR-21-5p mimics significantly promoted CaSki cell proliferation, migration and invasion, and inhibited cell apoptosis, whereas silencing of VHL counteracted these effects. Taken together, these results suggest that miR-21-5p may act as an oncogene by suppressing VHL expression in CC. To the best of our knowledge, the present study is the first to disclose this mechanistic and functional role of miR-21-5p in CC. A prior study suggested that tuberous sclerosis complex protein 1 expression is affected by VHL gene alterations and HIF- $1 \alpha$ expression in renal cell carcinoma (23). It was also demonstrated that miR-566 regulates vascular endothelial growth factor (VEGF) by targeting VHL in human glioblastoma in vitro and in vivo (24). Another study suggested that hsa-miR-331-3p downregulates VHL expression in hepatocellular carcinoma cell lines by targeting its 3'-UTR (25), and that miR-101 targets VHL to promote HIF-1 $\alpha$-mediated apoptosis and cell cycle arrest (26). Similarly, the inhibitory effects of miR-185 on cell proliferation and its activation of cell apoptosis by targeting VEGFA have been attributed to VHL inactivation in clear cell renal cell carcinoma (27). All these findings support the results of the present study and indicate the role of VHL, particularly the miR-21-5p/VHL axis, in tumorigenesis and cancer progression.

In summary, the present study provides novel evidence that the silencing of miR-21-5p inhibits the metastatic phenotype of CC cells by downregulating VHL. The results also suggest that miR-21-5p may be a novel therapeutic target for CC.

\section{References}

1. Sherris J, Herdman C and Elias C: Cervical cancer in the developing world. West J Med 175: 231-233, 2001.

2. Huang P, Xi J and Liu S: MiR-139-3p induces cell apoptosis and inhibits metastasis of cervical cancer by targeting NOB1. Biomed Pharmacother 83: 850-856, 2016.

3. Xu J, Zhang W, Lv Q and Zhu D: Overexpression of miR-21 promotes the proliferation and migration of cervical cancer cells via the inhibition of PTEN. Oncol Rep 33: 3108-3116, 2015.

4. Babashah S, Bakhshinejad B, Birgani MT, Pakravan K and Cho WC: Regulation of microRNAs by phytochemicals: A promising strategy for cancer chemoprevention. Curr Cancer Drug Targets: Jun 23, 2017 (Epub ahead of print).

5. Zhang H, Li T, Zheng L and Huang X: Biomarker MicroRNAs for diagnosis of oral squamous cell carcinoma identified based on gene expression data and MicroRNA-mRNA network analysis. Comput Math Methods Med 2017: 9803018, 2017.

6. Makiguchi T, Yamada M, Yoshioka Y, Sugiura H, Koarai A, Chiba S, Fujino N, Tojo Y, Ota C, Kubo H, et al: Serum extracellular vesicular miR-21-5p is a predictor of the prognosis in idiopathic pulmonary fibrosis. Respir Res 17: 110, 2016.

7. Han Y, Xu GX, Lu H, Yu DH, Ren Y, Wang L, Huang XH, Hou WJ, Wei ZH, Chen YP, et al: Dysregulation of miRNA-21 and their potential as biomarkers for the diagnosis of cervical cancer. Int J Clin Exp Pathol 8: 7131-7139, 2015.

8. Kowalczyk AE, Krazinski BE, Godlewski J, Grzegrzolka J, Kiewisz J, Kwiatkowski P, Sliwinska-Jewsiewicka A, Dziegiel P and Kmiec Z: SATB1 is down-regulated in clear cell renal cell carcinoma and correlates with miR-21-5p overexpression and poor prognosis. Cancer Genomics Proteomics 13: 209-217, 2016.

9. Zheng G, Li N, Jia X, Peng C, Luo L, Deng Y, Yin J, Song Y, Liu $\mathrm{H}, \mathrm{Lu} \mathrm{M}$, et al: MYCN-mediated miR-21 overexpression enhances chemo-resistance via targeting CADM1 in tongue cancer. J Mol Med (Berl) 94: 1129-1141, 2016.

10. Lopes-Ramos CM, Habr-Gama A, Quevedo Bde S, Felício NM, Bettoni F, Koyama FC, Asprino PF, Galante PA, Gama-Rodrigues J, Camargo AA, et al: Overexpression of miR-21-5p as a predictive marker for complete tumor regression to neoadjuvant chemoradiotherapy in rectal cancer patients. BMC Med Genomics 7: 68, 2014.

11. Park SK, Park YS, Ahn JY, Do EJ, Kim D, Kim JE, Jung K, Byeon JS, Ye BD, Yang DH, et al: MiR 21-5p as a predictor of recurrence in young gastric cancer patients. J Gastroenterol Hepatol 31: 1429-1435, 2016.

12. Bellissimo T, Russo E, Ganci F, Vico C, Sacconi A, Longo F, VitoloD, Anile M,DisioD, Marino M, et al: Circulating miR-21-5p and miR-148a-3p as emerging non-invasive biomarkers in thymic epithelial tumors. Cancer Biol Ther 17: 79-82, 2016.

13. Zhang J, Yao T, Wang Y, Yu J, Liu Y and Lin Z: Long noncoding RNA MEG3 is downregulated in cervical cancer and affects cell proliferation and apoptosis by regulating miR-21. Cancer Biol Ther 17: 104-113, 2016.

14. Kim WY and Kaelin WG: Role of VHL gene mutation in human cancer. J Clin Oncol 22: 4991-5004, 2004.

15. Kaelin WG Jr: The von Hippel-Lindau tumor suppressor gene and kidney cancer. Clin Cancer Res 10: 6290s-6295s, 2004.

16. Kaelin WG: The von Hippel-Lindau tumor suppressor protein: Roles in cancer and oxygen sensing. Cold Spring Harb Symp Quant Biol 70: 159-166, 2005.

17. Livak KJ and Schmittgen TD: Analysis of relative gene expression data using real-time quantitative PCR and the 2(-Delta Delta C(T)) method. Methods 25: 402-408, 2001.

18. Neal CS, Michael MZ, Rawlings LH, Van der Hoek MB and Gleadle JM: The VHL-dependent regulation of microRNAs in renal cancer. BMC Med 8: 64, 2010.

19. Liu X, Cai X, Hu B, Mei Z, Zhang D, Ouyang G, Wang J, Zhang W and Xiao W: Forkhead transcription factor 3a (FOXO3a) modulates hypoxia signaling via up-regulation of von Hippel-Lindau gene (VHL). J Biol Chem 291: 25692-25705, 2016.

20. Welford SM, Dorie MJ, Li X, Haase VH and Giaccia AJ: Renal oxygenation suppresses VHL loss-induced senescence that is caused by increased sensitivity to oxidative stress. Mol Cell Biol 30: 4595-4603, 2010.

21. Chetram MA, Bethea DA, Odero-Marah VA, Don-Salu-Hewage AS, Jones KJ and Hinton CV: ROS-mediated activation of AKT induces apoptosis via pVHL in prostate cancer cells. Mol Cell Biochem 376: 63-71, 2013. 
22. LaGory EL, Wu C, Taniguchi CM, Ding CC, Chi JT, von Eyben R Scott DA, Richardson AD and Giaccia AJ: Suppression of PGC-1 $\alpha$ is critical for reprogramming oxidative metabolism in renal cell carcinoma. Cell Rep 12: 116-127, 2015.

23. Damjanovic SS, Ilic BB, Beleslin Cokic BB, Antic JA, Bankovic JZ, Milicevic IT, Rodic GS, Ilic DS, Todorovic VN, Puskas $\mathrm{N}$ and Tulic CD: Tuberous sclerosis complex protein 1 expression is affected by VHL Gene alterations and HIF-1 $\alpha$ production in sporadic clear-cell renal cell carcinoma. Exp Mol Pathol 101: 323-331, 2016.

24. Xiao B, Zhou X, Ye M, Lv S, Wu M, Liao C, Han L, Kang C and Zhu X: MicroRNA566 modulates vascular endothelial growth factor by targeting Von HippelLandau in human glioblastoma in vitro and in vivo. Mol Med Rep 13: 379-385, 2016.
25. Cao Y, Zhang J, Xiong D, Wang D, Wu T, Huang A and Tang H: Hsa-miR-331-3p inhibits VHL expression by directly targeting its mRNA 3'-UTR in HCC cell lines. Acta Biochim Pol 62: 77-82, 2015.

26. Liu N, Xia WY, Liu SS, Chen HY, Sun L, Liu MY, Li LF, Lu HM, Fu YJ, Wang P, et al: MicroRNA-101 targets von Hippel-Lindau tumor suppressor (VHL) to induce HIF1 $\alpha$ mediated apoptosis and cell cycle arrest in normoxia condition. Sci Rep 6: 20489, 2016.

27. Ma X, Shen D, Li H, Zhang Y, Lv X, Huang Q, Gao Y, Li X, $\mathrm{Gu} \mathrm{L}$, Xiu S, et al: MicroRNA-185 inhibits cell proliferation and induces cell apoptosis by targeting VEGFA directly in von Hippel-Lindau-inactivated clear cell renal cell carcinoma. Urol Oncol 33: 169.e1-11, 2015. 